\title{
Node Synchronization for the Viterbi Decoder
}

\author{
GARY LORDEN, ROBERT J. MCELIECE, SENIOR MEMBER, IEEE, AND LAIF SWANSON
}

\begin{abstract}
Motivated by the needs of NASA's Voyager 2 mission, in this paper we describe an algorithm which detects and corrects losses of node synchronization in convolutionally encoded data. This algorithm, which would be implemented as a hardware device external to a Viterbi decoder, makes statistical decisions about node synch based on the hard-quantized undecoded data stream. We will show that in a worst-case Voyager environment, our method will detect and correct a true loss of synch (thought to be a very rare event) within several hundred bits; many of the resulting outages will be corrected by the outer Reed-Solomon code. At the same time, the mean time between false alarms is on the order of several years, independent of the signal-to-noise ratio.
\end{abstract}

\section{INTRODUCTION}

$\mathbf{R}$ ECENT studies [1], [2] have shown that at the very low signal-to-noise ratios NASA's Voyager 2 mission will encounter at Uranus in 1986, the performance of the ReedSolomion/Viterbi concatenated coding system could be seriously degraded by erratic behavior in the node synchronization subsystem of NASA's hardware decoder. In this paper we will show that this problem can be avoided by disabling the Viterbi decoder's internal synchronization hardware and replacing it with a simple outboard hardware node synchronizer whose details we describe in this paper. For definiteness, this paper will deal only with the system parameters relevant to Voyager, but our technique is applicable (with minor modifications) to any convolutionally encoded telecommunications system.

On Voyager, the high-rate downlink telemetry is protected by a $K=7$, rate $1 / 2$ convolutional code concatenated with a depth-4 interleaved $(255,223)$ Reed-Solomon code. In principle this combination provides excellent error protection (bit error probability $1.0 E-6$ ) for Voyager's highly sensitive imaging data, at bit signal-to-noise ratios as low as $2.9 \mathrm{~dB}$. Since the rate of the outer code is $223 / 255=-0.6 \mathrm{~dB}$, when the overall SNR is $2.9 \mathrm{~dB}$, the inner convolutional code is operating at about $2.3 \mathrm{~dB}$.

However, in practice, the performance of the concatenated system is significantly worse than theoretical predictions. One problem is carrier-loop jitter, which degrades performance by $0.5 \mathrm{~dB}$ or more [1]. This means that if the system bit SNR remains at the nominal $2.9 \mathrm{~dB}$ value, the inner convolutional code must operate at less than $2.0 \mathrm{~dB}$. This is a value much lower than that for which the Deep Space Network's hardware Viterbi decoders were designed. In this demanding environment, the Viterbi decoder's internal node synchronization hardware, whose function is to detect and correct true external losses of node synch, is prone to produce false alarms, i.e., spurious losses of node synch, and send useless data to the Reed-Solomon decoder until node synch is reestablished. In [2] it was shown that this hardware problem can degrade Voyager's performance by a further $1.0 \mathrm{~dB}$ or more.

Paper approved by the Editor for Communication Theory of the IEEE Communications Society for publication after presentation at the American Mathematical Society Summer Meeting, Toronto, Ont., Canada, August 1982. Manuscript received March 11, 1983; revised October 14, 1983. This work was supported by a contract from the National Aeronautics and Space Administration.

The authors are with the Jet Propulsion Laboratory, California Institute of Technology, Pasadena, CA 91109.
In this article we shall show that this data loss due to spurious node synch loss in the Viterbi decoder is completely avoidable. Our proposed solution involves disabling the Viterbi decoder's internal synchronization hardware and implementing an external node synch algorithm. Our algorithm is easy to implement and depends on likelihood calculations based on observations of the hard-quantized encoded data stream. In a worst-case Voyager environment, our method will detect and correct a true loss of node synch within several hundred bits; many of these outages will be corrected by the Reed-Solomon code. On the other hand, the mean time between false alarms for our technique (which is independent of the SNR) is on the order of several years. Thus, for practical purposes our technique introduces no false alarms, and the system SNR loss due to node synch problems will be eliminated, with no loss of protection against true node synch losses. As an outboard hardware device, our algorithm could be implemented on a single Deep Space Network standard single-board computer such as the iSBC $86 / 12$, at least at data rates up to $20 \mathrm{kbits} / \mathrm{s}$.

The paper is divided into three sections (this is Section I). In Section II, we present a functional description of our algorithm, together with a summary of the relevant mathematics. In Section III, we present some numerical performance results for our technique. They will quantify the assertions made above (mean time between false alarms, probability of uncorrectable errors due to true loss of synch, etc.). We also include in the Appendixes some background information.

\section{THE Up-Down COUNTER}

We adopt the following model, which has been found to be very accurate for coherent deep-space communication [4]. The information to be transmitted via the convolutional code (which in Voyager is already encoded) is a sequence $\cdots, M_{-4}$, $M_{-2}, M_{0}, M_{2}, M_{4}, \cdots$ of independent identically distributed random variables, each equally likely to be 0 or 1 . The encoded stream $\cdots, C_{-3}, C_{-2}, C_{-1}, C_{0}, C_{1}, \cdots$ is defined by the encoding equations $\overrightarrow{1}$

$$
\begin{gathered}
C_{2 k}=M_{2 k}+M_{2 k-2}+M_{2 k-4}+M_{2 k-6}+M_{2 k-12} \\
(\bmod 2) \\
C_{2 k-1}=M_{2 k}+M_{2 k-4}+M_{2 k-6}+M_{2 k-10}+M_{2 k-12}
\end{gathered}
$$

$(\bmod 2)$

We also define the \pm versions of the encoded stream:

$$
D_{j}= \begin{cases}+1 & \text { if } C_{j}=0 \\ -1 & \text { if } C_{j}=1\end{cases}
$$

The encoded bits $\left\{D_{k}\right\}$ are used to modulate a radio frequency signal, which is transmitted by Voyager to earth. After

1 We shall illustrate all of our results for the NASA standard $K=7$, rate $1 / 2$ convolutional code, but everything generalizes easily to any rate $1 / 2$ convolutional code. 
detection and demodulation, a sequence $\left\{\hat{D}_{k}\right\}$ is received, where $\hat{D}_{k}=D_{k}+Z_{k}$. The sequence $\left\{Z_{k}\right\}$ is the error sequence. If the noise process is additive white Gaussian noise, then the sequence $\left\{Z_{k}\right\}$ is i.i.d., the common distribution being Gaussian, mean zero and variance $\sigma^{2}=1 / \mu$, where $\mu$ is twice the symbol SNR.

The Viterbi decoder attempts to recover the message bits from the noisy code sequence $\left\{\hat{D}_{k}\right\}$. It does this by making a very efficient maximum likelihood estimate of each of the message bits $\left\{M_{2 j}\right\}$. However, in order to operate, the Viterbi decoder must have node synch, i.e., it must know which of the received symbols have even subscripts and which have odd subscripts. Of course, there are only two possibilities, but if the wrong hypothesis is made, the output of the Viterbi decoder will bear no useful relationship to the message stream $\left\{M_{2 j}\right\}$.

Our algorithm will provide node synch information for the Viterbi decoder. It is based on the hard-quantized received sequence $\left\{R_{k}\right\}$; where

$$
R_{k}= \begin{cases}0 & \text { if } \hat{D}_{k} \geqslant 0 \\ 1 & \text { if } \hat{D}_{k}<0\end{cases}
$$

Clearly $R_{k}=C_{k}+E_{k}(\bmod 2)$, where $\left\{E_{k}\right\}$ is i.i.d, and $E_{k}=$ 0 or 1 . The error probability $p_{e}=\operatorname{Pr}\left\{E_{k}=1\right\}$ is given by

$$
p_{e}=\frac{1}{\sqrt{2 \pi}} \int_{\mu}^{\infty} e^{-t^{2} / 2} d t
$$

where, as before, $\mu=2 E_{S} / N_{0}$.

Associated with the hard-quantized received sequence $\left\{R_{k}\right\}$ is the syndrome, or parity-check sequence $\left\{X_{k}\right\}[5]$ :

$$
\begin{aligned}
X_{k}= & R_{k}+R_{k-1}+R_{k-3}+R_{k-4}+R_{k-5}+R_{k-6} \\
& +R_{k-7}+R_{k-10}+R_{k-12}+R_{k-13} .
\end{aligned}
$$

If there are no errors, i.e., if $E_{k}=0$ for all $k$, then it follows from (1) and (2) that $X_{k}=0$ for all even subscripts $k$. In fact we have explicitly

$$
\begin{aligned}
X_{k}= & E_{k}+E_{k-1}+E_{k-3}+E_{k-4}+E_{k-5}+E_{k-6} \\
& +E_{k-7}+E_{k-10}+E_{k-12}+E_{k-13} \quad k \text { even. }
\end{aligned}
$$

For odd $\mathrm{k},(1)$ and (2) show that the $X_{k}$ 's are independent and take the values 0 and 1 with probability $1 / 2$ each, regardless of the $\left\{E_{k}\right\}$ sequence. (This is shown in detail in Appendix A.)

For even $k$, (5) shows that $X_{k}$ will be zero if and only if an even number of $\left\{E_{k}, E_{k-1}, E_{k-3}, E_{k-4}, E_{k-5}, E_{k-6}\right.$, $\left.E_{k-7}, E_{k-10}, E_{k-12}, E_{k-13}\right\}$ are one. The probability of this is easily seen to be

$$
\pi=\frac{1+\left(1-2 p_{e}\right)^{10}}{2} .
$$

The foregoing describes the distribution of the received sequence and of the parity-check sequence in case node synchronization is maintained. This will be called the in-synch hypothesis. The out-of-synch hypothesis describes the situation when node synchronization is in error. In this case the $R_{i}$ 's and $X_{i}$ 's behave as though the subscripts were shifted by one. Thus, under the out-of-synch hypothesis it is the odd parity checks that are correct with probability $\pi$ and the even ones that are purely random.

We assume that node synchronization has been acquired and maintained and that the in-synch hypothesis is initially true. We wish to monitor the received sequence so as to de- tect loss of synch, i.e., a sudden change making the received sequence obey the out-of-synch hypothesis. A method for doing this simply can be based on a general statistical technique [7] for detecting a change in distribution.:To apply this technique it is necessary to simplify the model by assuming that the parity checks $X_{i}$ are independent. They are not independent for even $i$, but the dependence between widely separated $X_{i}$ 's is slight, so calculations based on this assumption should be illustrative.

The method for detecting loss of synch is based on a counter with increments

$$
L\left(X_{n}\right)=\log \frac{q\left(X_{n}\right)}{p\left(X_{n}\right)}
$$

where $p\left(X_{n}\right)$ and $q\left(X_{n}\right)$ are the likelihoods of $X_{n}$ under the in-synch and out-of-synch hypotheses, respectively. As shown above

$$
p\left(X_{n}\right)= \begin{cases}1 / 2 & \text { if } n \text { is odd } \\ \pi & \text { if } n \text { is even and } X_{n}=0 \\ 1-\pi & \text { if } n \text { is even and } X_{n}=1\end{cases}
$$

whereas $q\left(X_{n}\right)$ reverses the odd and even cases. Thus,

$$
L\left(X_{n}\right)= \begin{cases}(-1)^{n+1} \log (2 \pi) & \text { if } X_{n}=0 \\ (-1)^{n+1} \log (2(1-\pi)) & \text { if } X_{n}=1 .\end{cases}
$$

The counter is defined by

$$
\begin{aligned}
& T_{0}=0 \\
& T_{n}=\max \left(\left(T_{n-1}+L\left(X_{n}\right)\right), 0\right), \quad n \geqslant 1 .
\end{aligned}
$$

A threshold $\gamma>0$ is chosen, and the process stops the first time $T_{n} \geqslant \gamma$. Since at this point there is a substantial likelihood ratio in favor of the out-of-synch hypotheses, the inference is made that loss of node synchronization has occurred. This loss of node synch can be remedied by either adding or deleting a channel symbol; the node synchronizer will alternate adding and deleting symbols in order to avoid ruining frame boundaries in case of false loss of synch.

The performance of such a counter for a particular $\gamma$ is characterized by two average run lengths (ARL's).

1) The short $A R L$. This is the average number of pairs of symbols needed to reach the threshold if the out-of-synch hypothesis is true from the beginning.

2) The long $A R L$. This is the average number of pairs of symbols needed to reach the threshold if the in-synch hypothesis remains true.

The short ARL gives an upper bound on the average time between loss of synch and its detection, since whenever loss of synch occurs, the counter has a nonnegative value. The long ARL (or its reciprocal) describes the frequency of false detection of loss of node synchronization.

An exact determination of the ARL's will be made in Section III, for a very slight modification of the scheme described. It is instructive, however, particularly for later comparisons, to consider their asymptotic behavior as $\gamma \rightarrow \infty$. It turns out that

$$
\text { short ARL } \sim \frac{\gamma}{I}
$$

and

$$
\text { long ARL } \sim \mathrm{Ce}^{\gamma} \text {, }
$$


so that

$$
\text { short ARL } \sim \frac{\log (\operatorname{long} \mathrm{ARL})}{I} .
$$

Here the constant $I$, which is much more critical than $C$, is the Kullback-Leibler information number per pair of symbols. This number is simply the average increment of the counter per pair of symbols when the out-of-synch hypothesis is true. Since each pair of symbols generates one even $\dot{X}_{n}$ and one odd $X_{n}$,

$$
\dot{I}=I_{1}+I_{2}
$$

where

$$
\begin{aligned}
I_{1} & =q(0 \mid n \text { odd }) \log 2 \pi+q(1 \mid n \text { odd }) \log (2(1-\pi)) \\
& =\pi \log (2 \pi)+(1-\pi) \log (2(1-\pi))
\end{aligned}
$$

and

$$
\begin{aligned}
I_{2} & =q(0 \mid n \text { even })(-\log 2 \pi)+q(1 \downarrow n \text { even })(-\log (2(1-\pi)) \\
& =-1 / 2 \log (4 \pi(1-\pi)) ;
\end{aligned}
$$

Table I shows the dependence of the information numbers on $p_{e}$, the symbol error probability. It should be noted that the information numbers decrease substantially as the symbol error probability becomes larger. Thus, the ARL's become less favorable as $p_{e}$ increases.

If one makes the very slight change of inspecting the counter only at even $n$, i.e., once for each pair of symbols, then a simpler description of the counter is possible. This is because two consecutive $\dot{X}_{n}$ 's $=0$ (successful parity checks) yield a net change of zero, as do consecutive $X_{n}$ 's $=1$. If, however, one of the pair of $X_{n}$ 's is 0 and the other 1 , then the total increment of the counter is easily seen to be $\pm \log (\pi / 1-\pi)$, with + if and only if the odd $X_{n}=0$ (i.e., both a successful check for the out-of-synch hypothesis and a failure for the in-synch hypothesis). Thus, the counter moves up and down by a fixed step size and standard random walk formulas [3, p. 351] can be used to derive exact formulas for the ARL's under the simplifying assumption of independence. Assuming without loss of generality that

$$
\gamma=m \log \frac{\pi}{1-\pi}
$$

$m$ an integer, one has

$$
\text { short ARL }=(\pi-0.5)^{-1}\left(m+\frac{1-(\pi /(1-\pi))^{-m}}{1-(\pi /(1-\pi))}\right)
$$

and

$$
\text { long } \mathrm{ARL}=(\pi-0.5)^{-1}\left(\frac{(\pi /(1-\pi))^{m}-1}{1-(1-\pi) / \pi}-m\right)
$$

Table II illustrates the relationship between the two ARL's as a function of $m$ for two symbol error probabilities, 0.08 and 0.1 .

\section{Counter with Memory}

A simple parity check counter does a fairly good job of node synchronization in case of high SNR [5]. But in the case of high symbol error probability, the probability of parity
TABLE I

DEPENDENCE OF INFORMATION NUMBERS ON SYMBOL ERROR PROBABILITY

\begin{tabular}{ccclll}
\hline$p$ & \multicolumn{1}{c}{$\pi$} & \multicolumn{1}{c}{$I_{1}$} & \multicolumn{1}{c}{$I_{2}$} & \multicolumn{1}{c}{$I$} \\
\hline 0.08 & 0.5875 & 0.0154 & 0.0155 & 0.0309 \\
0.10 & 0.5537 & $5.78 \times 10^{-3}$ & $5.80 \times 10^{-3}$ & 0.0116 \\
0.12 & 0.5321 & $2.07 \times 10^{-3}$ & $2.07 \times 10^{-3}$ & $4.14 \times 10^{-3}$ \\
\hline
\end{tabular}

TABLE II

RELATIONSHIP BETWEEN AVERAGE RUN LENGTHS

\begin{tabular}{cccccc}
\hline$p$ & $\dot{n}$ & $f$ & $m$ & Short ARL & Long ARL \\
\hline 0.1 & 0.5537 & 0.01157 & 10 & 118 & 547 \\
& &. & 15 & 205 & 2,062 \\
& & & 20 & 296 & 6,693 \\
0.08 & 0.5875 & 0.03091 & 10 & 88 & 1,164 \\
& & & 15 & 145 & 7,496 \\
& & & 20 & 202 & 44,850 \\
\hline
\end{tabular}

check error is quite high. For example, symbol error probability 0.1 corresponds to $\pi=0.45$. Thus; in-synch data with $p_{e}=0.1$ will fail an even parity check with probability 0.45 , while out-of-synch data will fail the even parity checks with probability 0.5 . A counter to distinguish between distributions which are so close will either require a long time to react to incorrect synch or have a large probability of incorrect change. Performance numbers for such a counter are indicated in Table II above:

If the parity checks were independent, there would be no way to improve this performance [7]. But the parity checks are not independent. This is because, under the in-synch hypothesis, one channel symbol error changes the value of five even parity checks. Under the in-synch hypothesis, for example, an isolated error in the $(n-12)$ th channel symbol will cause parity check failures at time $n, n-2, n-6, n-8$, and $n-$ 12. Thus, a long sequence of successful parity checks would lead us to believe that another success is on the way, while the sequence $X_{n-12}=1, X_{n-10}=0, X_{n-8}=1, X_{n-6}=1$, $X_{n-4}=0 ; \bar{X}_{n-2}=1$ would lead one to believe that $X_{n}$ is very likely to be 1 . The reason a log-likelihood counter works so well in the independent case is that by adding logarithms we multiply their arguments. After receiving parity checks $X_{1}=$ $x_{1}, X_{2}=x_{2}, \cdots, X_{n}=x_{n}$, where each $x_{i}$ is 0 or 1 , the counter contains

$$
=\log \frac{\prod_{i=1}^{n} q\left(x_{i}\right)}{\prod_{i=1}^{n} p\left(x_{i}\right)}
$$$$
\log \frac{q\left(x_{1}\right)}{p\left(x_{1}\right)}+\log \frac{q\left(x_{2}\right)}{p\left(x_{2}\right)}+\cdots+\log \frac{q\left(x_{n}\right)}{p\left(x_{n}\right)}
$$

If the parity checks were independent, this would be exactly

$$
\log \left(\frac{q\left(X_{1}=x_{1}, X_{2}=x_{2}, \cdots, X_{n}=x_{n}\right)}{p\left(X_{1}=x_{1}, X_{2}=x_{2}, \cdots, X_{n}=x_{n}\right)}\right),
$$

the $\log$ of the ratio of the likelihood of the string $\left(X_{1}=x_{1}\right.$, 
$\left.X_{2}=x_{2}, \cdots, X_{n}=x_{n}\right)$ under the two hypotheses, which is statistically optimal for detecting loss of synchronization.

In the case of noisy convolutionally encoded data, the probability of a string of parity checks is not just the product of the probabilities of the individual parity checks. Updating the $p$ probability of a string requires the conditional probability $p\left(X_{n}=x_{n} \mid X_{n-1}=x_{n-1}, X_{n-2}=x_{n-2}, \cdots, X_{1}=\right.$ $\left.x_{1}\right)$. Therefore, a counter with increment

$$
\log \left(\frac{q\left(X_{n}=x_{n} \mid X_{n-1}=x_{n-1}, \cdots, X_{1}=x_{1}\right)}{p\left(X_{n}=x_{n} \mid X_{n-1}=x_{n-1}, \cdots, X_{1}=x_{1}\right)}\right)
$$

In general, the counter using $k$ parity checks has increment

$\log \left(\frac{q\left(X_{n}=x_{n} \mid X_{n-2}=x_{n-2}, \cdots, X_{n-2 k+2}=x_{n-2 k+2}\right)}{p\left(X_{n}=x_{n} \mid X_{n-2}=X_{n-2}, \cdots, X_{n-2 k+2}=x_{n-2 k+2}\right)}\right)$.

The system which we have investigated in detail is therefore a system which takes hard-quantized received channel symbols $R_{1}, R_{2}, \cdots$, creates parity checks $X_{14}, X_{15}, \cdots$ with $X_{i}=R_{i}+$ $R_{i-1}+R_{i-3}+R_{i-4}+R_{i-5}+R_{i-6}+R_{i-7}+R_{i-10}+$ $R_{i-12}+R_{i-13}$, and keeps a counter whose increment at time $n$ is

$$
\log \left(\frac{q\left(X_{n}=x_{n} \mid X_{n-2}=x_{n-2}, X_{n-4}=x_{n-4}, \cdots, X_{n-2 k+2}=x_{n-2 k+2}\right)}{p\left(X_{n}=x_{n} \mid X_{n-2}=x_{n-2}, X_{n-4}=x_{n-4}, \cdots, X_{n-2 k+2}=x_{n-2 k+2}\right)}\right) .
$$

would, after step $r$, contain

$$
\begin{gathered}
\sum_{n=1}^{r} \log \frac{q\left(X_{n}=x_{n} \mid X_{n-1}=x_{n-1}, \cdots, X_{1}=x_{1}\right)}{p\left(X_{n}=x_{n} \mid X_{n-1}=x_{n-1}, \cdots, X_{1}=x_{1}\right)} \\
=\log \frac{\prod_{n=1}^{r} q\left(X_{n}=x_{n} \mid X_{n-1}=x_{n-1}, \cdots, X_{1}=x_{1}\right)}{\prod_{n=1}^{r} p\left(X_{n}=x_{n} \mid X_{n-1}=x_{n-1}, \cdots, X_{1}=x_{1}\right)} \\
=\log \frac{q\left(X_{r}=x_{r}, X_{r-1}=x_{r-1}, \cdots, X_{1}=x_{1}\right)}{p\left(X_{r}=x_{r}, X_{r-1}=x_{r-1}, \cdots, X_{1}=x_{1}\right)}
\end{gathered}
$$

exactly the $\log$ of the quantity we desire. Of course, a real counter will not take into account a past of indefinite length. But a (possibly large) integer $m$ can be chosen, and a counter constructed whose increment at time $n$ is

$$
\log \left(\frac{q\left(X_{n}=x_{n} \mid X_{n-1}=x_{n-1}, \cdots, X_{n-m}=x_{n-m}\right)}{p\left(X_{n}=x_{n} \mid X_{n-1}=x_{n-1}, \cdots, X_{n-m}=x_{n-m}\right)}\right) .
$$

As might be expected, for large $m$, the information number approaches the information number for the hypothetical counter based on the indefinite past of the parity check sequence. This is verified in Appendix A. Moreover, the information numbers obtained using the parity check sequence are identical with information numbers obtainable from the hard quantized received sequence. This means that there is no loss of information or efficiency in using the parity check sequence instead of the hard quantized received sequence to detect loss of node synchronization-this is also shown in Appendix A. Also, Appendix A shows that the counter increments depend only on parity checks of the same (odd or even) type, i.e., (8) is unchanged if the given $\left(X_{n-1}=x_{n-1}, X_{n-2}=\right.$ $\left.x_{n-2}, \cdots\right)$ is replaced by $\left(X_{n-2}=x_{n-2}, X_{n-4}=x_{n-4}, \cdots\right)$.

We will describe the counter in terms of the number of parity checks used to determine the counter increment, and we will call this $k$. For example, the "simple" (memoryless) parity check counter corresponds to $k=1$, while the counter for $k=8$ has counter increments

$$
\log \left(\frac{q\left(X_{n}=x_{n} \mid X_{n-2}=x_{n-2}, \cdots, X_{n-14}=x_{n-14}\right)}{p\left(X_{n}=x_{n} \mid X_{n-2}=x_{n-2}, \cdots, X_{n-14}=x_{n-14}\right)}\right) .
$$

In order to design this counter, we need to know $p\left(X_{n}=\right.$ $\left.x_{n} \mid X_{n-2}=x_{n-2}, \cdots, X_{n-2 k+2}=x_{n-2 k+2}\right)$ and $q$ of the same event for even and odd $n$. To calculate these values, remember that for in-synch data, the even parity checks depend only on the sequence of channel symbol errors. So given a probability $p_{e}$ of symbol error, we can calculate the probability that $E_{n}=e_{n}, E_{n-1}=e_{n-1}, \cdots, E_{n-2 k-11}=e_{n-2 k-11}$ for each sequence $e_{n}, e_{n-1}, \cdots, e_{n-2 k-11}$, and use these probabilities to calculate $p\left(X_{n}=x_{n}, \cdots, X_{n-2 k+2}=x_{n-2 k+2}\right)$ and $p\left(X_{n-2}=x_{n-2}, \cdots, X_{n-2 k+2}=x_{n-2 k+2}\right)$ and find $p\left(X_{n}=x_{n} \mid X_{n-2}=x_{n-2}, \cdots, X_{n-2 k+2}=x_{n-2 k+2}\right)$ for even $n$.

For odd $n$, just as in the case of the simple counter, the $X_{n}$ 's are independent with $p\left(X_{n}=0\right)=1 / 2$. Thus

$$
\begin{aligned}
& p\left(X_{n}=x_{n}+X_{n-2}=x_{n-2}, \cdots, X_{n-2 k+2}=x_{n-2 k+2}\right) \\
& =1 / 2 \quad \text { for odd } n .
\end{aligned}
$$

To calculate the probabilities $q$ associated with the out-ofsynch hypothesis, just exchange even with odd in the above argument. From these values, we can calculate the counter increments. We did this assuming a channel symbol error rate of 0.1 , which corresponds to a Viterbi decoded bit error rate of $5 \times 10^{-3}$, the standard for imaging data. Voyager's data rate has been adjusted so that this is the largest channel symbol error rate which will be encountered.

\section{Information Numbers and Run Lengths}

Just as in the case of the up-down counter, the average run lengths are essentially determined by the threshold $\gamma$ and the information number $I$, i.e., the average increment of the counter when the data are truly out of synch. For large $\gamma$ the run lengths are approximately

$$
\text { short ARL } \sim \frac{\gamma}{I}
$$

and

$$
\text { long } \mathrm{ARL} \sim C e^{D \gamma}
$$

where $C, D$, and $I$ depend, of course, on $k$, the number of parity checks used in determining the counter increment. These approximations were borne out by the simulations reported in Section III, and the constants $C$ and $D$ were determined empirically for each $k$ considered. $I$, the information number, is the average increment of the counter when the data are truly out of synch. (Of course, $I$ depends upon $k$, the number of parity checks used in determining the counter increment, and is an increasing function of it.) 
TABLE III

INFORMATION NUMBERS

\begin{tabular}{rccc}
\hline \multicolumn{1}{c}{$k$} & Total & Out-of-sync & In-sync \\
\hline 16 & 0.0862 & 0.0601 & 0.0261 \\
8 & 0.0633 & 0.0406 & 0.0228 \\
\hline
\end{tabular}

The information $I$ from a pair of parity checks is the sum of the information number $I_{1}$ from the odd parity checks (the average increment of the counter at odd $n$ ) and $I_{2}$ from the even parity checks. Table III shows the contributions of these two parity check subsequences, revealing that the odd checks contribute roughly two thirds of the total information.

In designing the detection algorithm, we must choose a symbol error probability $p_{e}$, at which the counter is designed to operate most efficiently. In practice, as the true symbol error probability varies/with the signal-to-noise ratio, it will generally be smaller than $p_{e}$, so that fewer parity check failures occur. In this case, the counter will perform better whether the data are in synch or not, i.e., the short ARL will be reduced and the long ARL increased.

In case the signal-to-noise ratio is degraded so much that the symbol error probability exceeds $p_{e}$, however, a problem arises in the performance of the counter, as both the short and long ARL's become less favorable. In real life, the symbol error probability is not constant, and the fact that the long ARL's shorten during periods of low signal-to-noise ratio would introduce the same spurious loss of synch which seems to plague the current Viterbi decoders. If the short ARL gets longer during a period of high symbol error rate, this causes the response time to a loss of synch to increase and may cause a string of data to be lost if a true loss of synch hits at the time of low signal-to-noise ratio, but a shortening of the long ARL whenever there is such a period will have a far greater effect on the overall behavior of the system.

This degradation of long ARL can be totally eliminated by giving up the information $I_{2}$ from the even parity check subsequence. When the counter uses only the odd subsequence, the long ARL is unaffected by the signal-to-noise ratio. This is because the odd checks are completely random (independent, with 50 percent probability of success) whenever the process is in synch.

Since most of the information comes from the odd subsequence anyway, we believe it is prudent to base the counter on this subsequence alone. The performance parameters in the next section were all calculated for counters based solely on the out-of-synch parity checks. It should be repeated, however, that these performance figures are based on the assumption that the data are a sequence of i.i.d. random variables equally likely to be \pm 1 . If the actual data stream deviates significantly from this model, e.g., if long strings of zeros or ones are probable, then the performance of our algorithm will not be as good as predicted.

\section{Performance Numbers}

The size of the ROM needed for counter increments is $2^{k}$. As before, we will describe the counter in terms of the number $k$ of parity checks needed to compute the counter increment.

Once $k$ is chosen, the log-likelihood scheme determines the counter increments. The only question left in algorithm design is the threshold at which the system is declared out of synch. If the threshold is high, the probability of false loss of synch is low, or equivalently, the time between false losses of synch is long. On the other hand, a high threshold will also make the short run length (or the time between loss of synch and detection of that loss of synch) large.

We first consider the influence of short run length on system performance. There is an obvious reason to want the short run length to be small: the sooner after a loss of synch that the system gets back on track, the better. But there is another reason as well. Voyager has a concatenated coding scheme: after the convolutional code is Viterbi decoded, an additional code, the Reed-Solomon code, is decoded. As far as the ReedSolomon decoder is concerned, the data stream during the short run is just a stream of bad data. (Of course, if the out-ofsynch condition was caused by the deletion of a symbol, and the node-synchronizer solves the problem by deleting another symbol, then a whole bit has been deleted, and frame boundaries are lost as well. In this case, the data during the short run can never be recovered. So we will consider the case in which the total number of channel symbols has not been changed. This will be true when the out-of-synch condition was caused by a spurious loss of synch caused by the node synchronizer, since the synchronizer will alternate adding and deleting channel symbols, and will be true half of the time anyway.) The Reed-Solomon decoder can recover a fair amount of bad data, and so if the short run is short enough, the Reed-Solomon decoder will be able to recover it most of the time. So the length of the average short run is not as important as the probability that the Reed-Solomon decoder will be able to recover the data in the short run. For the $k=8$ counter, assuming Viterbi burst statistics for $2.3 \mathrm{~dB}$ [8] and depth 4 interleaved Reed-Solomon words, Table IV shows the probability of decoding for a word in a frame wholly containing a short run.

This same table shows the long run lengths (both in bits and in time, at the reasonable Voyager data rate of $20000 \mathrm{bits} / \mathrm{s}$ ) for these same thresholds. Looking, for example, at threshold 15 , we see that the probability that a word contained in a short run will be corrected by the Reed-Solomon decoder is $2 / 3$, and that synch is lost incorrectly every two days.

Can we do better? In fact, by going to $k=16$, we can do much better. Table $\mathrm{V}$ shows this same information with $k=$ 16. With $k=16$ and threshold 14.5, a word which is in a frame attacked by a short run will decode correctly with probability 0.86 , and the mean time between false losses of synch (average long run time) is 6.7 years.

These numbers were obtained by simulation methods explained in Appendix B

Several other questions can be asked about the performance of the counter. What if a short run hits more than one frame? With $k=16$ and threshold 14.5 , the probability that a short run hits more than one frame is 0.033 . And even if the short run does intersect more than one frame, the probability that it causes a word error in each frame is less than 0.005 . Thus, the probability of a decoder error in each of two consecutive frames because of a spurious loss of node synchronization is less than 0.0002 .

Another question is the probability of some loss of data due to a short run. We saw that in the case $k=16$, threshold 14.5 , the probability that any one word fails to decode is 0.14 , but since decoding failures in the four words are by no means independent, this does not tell us the probability that there is some loss of data-that is, that one or more of the four interleaved Reed-Solomon words fails to decode. In the case $k=16$, threshold 14.5 , this probability is 0.19 .

This counter is "tuned" to the channel; that is, counter increments are based on probabilities, calculated from the channel model, of each block under the "in-synch hypothesis" and the "out-of-synch hypothesis." To make a counter for a channel with other than independent errors, one would make the same calculations using probabilities for that channel. 
TABLE IV

COUNTER PERFORMANCE WITH $K=8$

\begin{tabular}{|c|c|c|c|c|c|c|}
\hline \multirow{2}{*}{ Threshold } & \multirow{2}{*}{$\begin{array}{c}\text { Mean Short Run } \\
\text { (bits) }\end{array}$} & \multirow{2}{*}{\multicolumn{2}{|c|}{$\begin{array}{l}\text { Probab1lity that a } \\
\text { Reed-Solomon Word } \\
\text { In a Short Run W111 Decode }\end{array}$}} & \multicolumn{3}{|c|}{ Mean Long Run } \\
\hline & & & & Bits & Time & (at 20,000 bps) \\
\hline 5 & 116.6 & $\$$ & .98 & $6.1 \times 10^{4}$ & & 3 seconds \\
\hline 10 & 238.6 & & .86 & $5.2 \times 10^{7}$ & & 43 minutes \\
\hline 15 & 363.3 & & .66 & $4.5 \times 10^{10}$ & & 26 days \\
\hline 16 & 387.6 & & .62 & $1.7 \times 10^{11}$ & & 100 days \\
\hline 18 & 438.0 & & .52 & $2.6 \times 10^{12}$ & & 4 years \\
\hline 20 & 487.4 & & .43 & $3.8 \times 10^{13}$ & & 60 years \\
\hline
\end{tabular}

TABLE V

COUNTER PERFORMANCE WITH $K=16$

\begin{tabular}{|c|c|c|c|c|}
\hline \multirow[b]{2}{*}{ Threshold } & \multirow{2}{*}{$\begin{array}{c}\text { Mean Short Run } \\
\text { (bits) }\end{array}$} & \multirow{2}{*}{$\begin{array}{l}\text { Probability that a } \\
\text { Reed-Solomon Word } \\
\text { in a Short Run will Decode }\end{array}$} & \multicolumn{2}{|c|}{ Mean Long Run } \\
\hline & & & Bits & Time (at 20,000 bps) \\
\hline 5 & 88.6 & .99 & $5.9 \times 10^{6}$ & 5 minutes \\
\hline 10 & 171.4 & .95 & $7.9 \times 10^{9}$ & 4 days \\
\hline 11.5 & 196.4 & .93 & $6.0 \times 10^{10}$ & 35 days \\
\hline 13 & 221.7 & .90 & $5.0 \times 10^{11}$ & 290 days \\
\hline 14.5 & 246.9 & .87 & $4.2 \times 10^{12}$ & 6.7 years \\
\hline 18 & 305.1 & .77 & $6.1 \times 10^{14}$ & 960 years \\
\hline 20 & 338.8 & .71 & $1.0 \times 10^{16}$ & 16,000 years \\
\hline
\end{tabular}

\section{APPENDIX A}

\section{PROOFS}

This Appendix gives mathematical proofs of the statements made in Section II.

We use the same random processes to model the situation: 1) $\cdots, M_{-2}, M_{0}, M_{2}, \cdots$ an i.i.d. process, $P\left(M_{0}=0\right)=P\left(M_{0}=\right.$ 1) $=1 / 2$, representing the message; 2) $\cdots, E_{-2}, E_{-1}, E_{0}, E_{1}$, ‥ an i.i.d. process, $P\left(E_{0}=1\right)=$ symbol error probability, representing the errors in the received sequence and independent of the sequence $\left.\cdots, M_{-2}, M_{0}, M_{1}, \cdots, 3\right) \cdots, R_{-2}, R_{-1}, R_{0}$, $R_{1}, \cdots$, the convolutionally encoded $M_{i}$ 's added to the $E_{i}$ 's, representing the hard-quantized received channel symbols; and 4) $\cdots, X_{-1}, X_{0}, X_{1}, \cdots$ the sequence of parity checks derived from the $R_{i}$ 's.

Proposition: $p\left(X_{n}=x_{n}, X_{n-1}=x_{n-1}, \cdots, X_{n-2 k}=\right.$ $\left.x_{n-2 k}\right)=\left[p\left(X_{n}=x_{n}, X_{n-2}=x_{n-2}, \cdots, X_{n-2 k}=x_{n-2 k}\right) \cdot\right.$ $\left.p\left(X_{n-1}=x_{n-1}, \cdots, X_{n-2 k+1}=x_{n-2 k+1}\right)\right]$

Proof: First observe that for $m$ odd,

$$
p\left(X_{m}=1 \mid X_{m-2}, X_{m-4}, \cdots, \cdots E_{-1}, E_{0}, E_{1}, \cdots\right)=1 / 2
$$

because $X_{m}$ is a sum of $M_{m+1}$ and other variables, $p\left(M_{m+1}=\right.$ 1) $=1 / 2$, and $M_{m+1}$ is independent of all the random variables on which we are conditioning. This means that for odd $m$

$$
\begin{aligned}
p\left(X_{m}=x_{m}, X_{m-2}\right. & =x_{m-2}, \cdots, \\
X_{m-2 l} & \left.=x_{m-2 l} \mid \cdots, E_{-1}, E_{0}, E_{1}, \cdots\right) \\
& =2^{-l-1} \quad \text { a.s. }
\end{aligned}
$$

for any sequence $\left(x_{m}, x_{m-2}, \cdots, x_{m-2 l}\right)$ of zeros and ones. But the values of the even parity checks are determined entirely by $\cdots, E_{-1}, E_{0}, E_{1}, \cdots$, and so are independent of the odd parity checks.

Notice: 1) The fact that we looked at a sequence of odd length was convenient for notation but had no effect on the proof. 2) We showed not only that the even and odd parity checks are independent, but that the odd parity checks are themselves i.i.d. with probability $1 / 2$ of success. 3 ) The same result holds for the measure $q$, reversing the roles of even and odd.

Corollary: $p\left(X_{n}=x_{n} \mid X_{n-1}, X_{n-2}, \cdots\right)=p\left(X_{n}=x_{n} \mid\right.$ $\left.X_{n-2}, X_{n-4}, \cdots\right)$, and the same for $q$. If $n$ is odd, $p\left(X_{n}=\right.$ $\left.x_{n} \mid X_{n-2}, X_{n-4}, \cdots\right)=1 / 2$; for even $n, q\left(X_{n}=x_{n} \mid X_{n-2}\right.$, …) $=1 / 2$.

Definition: The one-sided, pseudoparity check sequence $\bar{X}_{1}, \bar{X}_{2}, \cdots$ is the parity check sequence based on the process $\cdots, 0,0, R_{1}, R_{2}, R_{3}, \cdots$. That is, $\bar{X}_{1}=R_{1}, \bar{X}_{2}=R_{2}+R_{1}$, $\bar{X}_{3}=R_{3}+R_{2}, \cdots, \bar{X}_{n}=X_{n}$ for $n \geqslant 14$.

Proposition: Every event of the form $\left(\bar{X}_{1}=x_{1}, \bar{X}_{2}=x_{2}\right.$, $\left.\cdots, \vec{X}_{n}=x_{n}\right)$ corresponds to exactly one event $\left(R_{1}=r_{1}, \cdots\right.$, $R_{n}=r_{n}$ ).

Proof: The $\bar{X}_{i}$ 's are derived from the $R_{i}$ 's. Going backwards, knowing $\bar{X}_{1}$ tells you $R_{1}$, and knowing $\bar{X}_{i}$ and $R_{1}, \cdots$, $R_{i-1}$ tells you $R_{i}$.

Corollary: A log-likelihood counter based on the $\bar{X}_{i}$ 's will always contain exactly the same value as one based on the $R_{i}$ 's.

Proposition: If $I_{m}$ is the information number of any loglikelihood counter with inputs based on the last $m$ outputs 
of any discrete random process and $I$ the information number with increments based on the indefinite past, then $\lim _{m \rightarrow \infty} I_{m}=$ I.

Proof: For simplicity, we give the proof for random variables taking on the values 0 and 1 . The proof for random variables taking on finitely many values is exactly the same.

Let $\cdots, Y_{-n}, \cdots, Y_{0}, Y_{1}, \cdots$ be the stationary process. (In our case, the $Y_{i}$ 's are pairs of hard-quantized received channel symbols.) Let

$$
f_{n}(y, j)=\log \left(\frac{\left(p\left(Y_{0}=j \mid Y_{-1}, Y_{-2}, \cdots, Y_{-n}\right)\right)(y)}{\left(q\left(Y_{0}=j \mid Y_{-1}, Y_{-2}, \cdots, Y_{-n}\right)\right)(y)}\right)
$$

where $y$ is chosen from the probability space on which the $Y_{i}$ 's are defined and $j$ is 0 or 1 .

It is a standard result of martingale theory [10, p. 237] that

$$
\begin{aligned}
\lim _{n \rightarrow \infty} f_{n}(y, j) & =f(y, j) \\
& =\log \left(\frac{\left(p\left(Y_{0}=j \mid Y_{-1}, \cdots\right)\right)(y)}{\left(q\left(Y_{0}=j \mid Y_{-1}, \cdots\right)\right)(y)}\right), \text { a.s. }
\end{aligned}
$$

and so by the dominated convergence theorem

$$
\lim _{n \rightarrow \infty} \int f_{n}\left(y, Y_{0}(y)\right) d p=\int f\left(y, Y_{0}(y)\right) d p
$$

and the same for integration with respect to $q$. But these are just the information numbers for the counters.

Notice: A log-likelihood counter using $k$ parity checks based on parity checks $\bar{X}$ will, after time $2 k+14$, have exactly the same increments as a log-likelihood counter using $k$ parity checks and based on parity checks $X$, because the values of $\bar{X}$ and $X$ are exactly the same starting at time 14 .

Theorem: As $k$ approaches infinity, the information in the log-likelihood counter whose increment at time $n$ is

$$
\log \frac{p\left(X_{n}=x_{n} \mid X_{n-2}, \cdots, X_{n-2 k+2}\right)}{q\left(X_{n}=x_{n} \mid X_{n-2}, \cdots, X_{n-2 k+2}\right)}
$$

kept separately for $n$ even and odd, approaches all the synch information in the hard-quantized received channel symbol stream $R_{1}, R_{2}, \cdots$.

Proof: As $k$ goes to infinity, the information in a counter $C(1)$ based on past of length $2 k$ of the hard-quantized received channel stream approaches all the information in the stream. The values in $C(1)$ are exactly the same as would be in a counter $C(2)$ based on the past of length $2 k$ of the (nonstationary) parity checks $\bar{X}_{i}$. For $n>2 k+14$, the increments in counter $C(2)$ are the same as those in a counter $C(3)$ based on the probabilities of $\left(X_{n} \mid X_{n-1}, \cdots, X_{n-2 k}\right)$. But, since even and odd parity checks are independent, this is exactly the same as the counter of the theorem.

\section{APPENDIX B}

\section{Simulation OF ARL's}

The performance figures of Section III were obtained by simulation. The short ARL's were simulated directly by generating independent symbol errors with probability $p$, computing the resulting parity check stream, and feeding it to the counter with threshold $\gamma$. Direct simulation of the long ARL's is not feasible, however, because (as the results show) the time required to generate a statistically useful sample of long run lengths would be too great.
The long ARL's were simulated by a modification of a standard technique called "importance sampling" [9], in which the process to be analyzed-in this case, the parity check sequence-. is generated using a probability distribution $Q$ different from the distribution $P$ for which results are desired. In our case, $P$ specifies independent 50-50 results for the out-of-synch parity checks-so that the time for the counter to reach a distant threshold $\gamma$ is quite large. A distribution $Q$ was chosen to make the counter reach the threshold more quicklynamely, independent parity checks with probability of failure $\pi^{*}$, substantially less than $1 / 2$.

The method of importance sampling is based on the simple fact that for any event $A$, the probability of $A$ under $P$ can be obtained from simulations carried out under the distribution $Q$. The key is provided by the identity

$$
P(A)=\int_{A} d P=\int_{A}\left(\frac{d P}{d Q}\right) d Q .
$$

Here the quantity $d P / d Q$ is the Radon-Nikodym derivative of $P$ with respect to $Q$, which in our application is simply the likelihood ratio

$$
\frac{P\left(X_{1}\right) \cdots P\left(X_{n}\right)}{Q\left(X_{1}\right) \cdots Q\left(X_{n}\right)}
$$

of the parity checks $X_{1}, \cdots, X_{n}$ up to the time that $A$ occurs. Since $P\left(X_{i}\right) \equiv 1 / 2$, and $Q\left(X_{i}\right)=\pi^{*}$ if $X_{i}=1$ (failure), $=1-$ $\pi^{*}$ if $X_{i}=0$ (success), relation (1) can be made more explicit. Using $E_{Q}$ to denote expectation under $Q$, it takes the form

$$
P(A)=E_{Q}\left[\left(\frac{1}{2 \pi^{*}}\right)^{F}\left(\frac{1}{2\left(1-\pi^{*}\right)}\right)^{S} 1\{A\}\right]
$$

where $F$ and $S$ are the numbers of failures and successes, respectively, in the out-of-synch parity checks up to the time that $A$ occurs, and $1\{A\}=1$ if $\ddot{A}$ occurs, $=0$ otherwise.

The simulation of the long ARL was based on the definition of a counter cycle. Starting from a given state $s^{*}$ of $k$ zeros and ones, the cycle ends the first time that the counter resets to zero with the same sequence $s^{*}$ in its memory. Let $T$ denote the time (number of symbol pairs) for a cycle to end and let $N$ denote the number of cycles until the threshold $\gamma$ is crossed. Then using a standard result called Wald's equation [3, vol. II, p. 601], we have

$$
\text { long } \mathrm{ARL}=E N \cdot E T \text {. }
$$

(Actually, the right side gives the expected time until the end of the first cycle on which $\gamma$ is crossed, but the extra time to end the cycle after crossing is negligible compared to the long ARL.) The quantity $E T$ was easily simulated directly, since when the parity checks are random the cycles end fairly quickly (and do not depend on $\gamma$ at all). The evaluation of $E N$ was based on

$$
E N=\frac{1}{P(A)}
$$

where $A=\{\gamma$ is crossed $\}$ for a given cycle, and $P(A)$ was simulated using the method of importance sampling, as described above.

Importance sampling was used to estimate $P(A)$ in several independent sets of simulations. Not surprisingly, the estimates were more stable for smaller thresholds. After all the data were gathered, a least squares line was drawn through the points representing small thresholds (see Figs. 1 and 2). These lines were used for the long ARL's in Tables IV and V. 


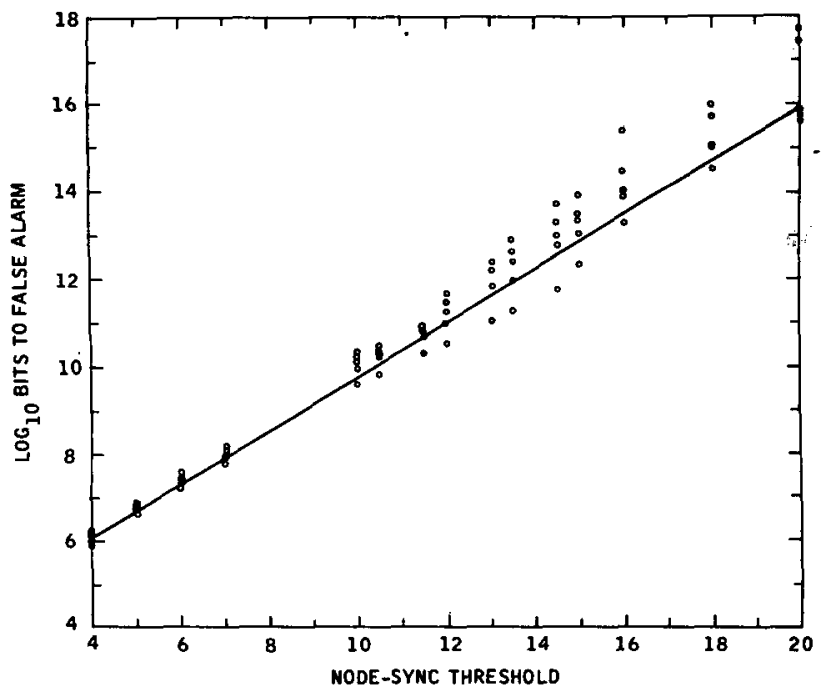

Fig. 1. Mean time to false alarm, $k=16$; least squares line from thresholds 4 , 5,6 , and 7 .

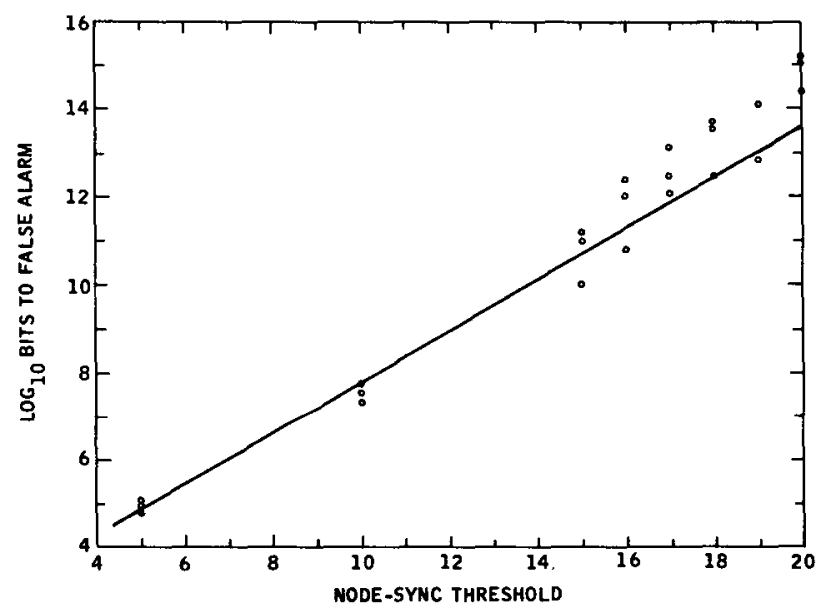

Fig. 2. Mean time to false alarm, $k=8$; least squares line from thresholds 5 , 10 , and 15 .

\section{REFERENCES}

[1] L. J. Deutsch and R. L. Miller, "The effects of Viterbi decoder node synchronization losses on the telemetry receiving system," Jet Propulsion Lab., Pasadena, CA, TDA Progress Rep. 42-68, Aug. 15, 1981.

[2] —-, "Viterbi decoder node synchronization losses in the Reed-Solomon/Viterbi concatenated channel," Jet Propulsion Lab., Pasadena, CA, TDA Progress Rep. 42-71, Nov. 15, 1982.

[3] W. Feller, An Introduction to Probability Theory and its Applications, vols. I and II. New York: Wiley, 1968, 1971.

[4] S. Golomb et al., Digital Communications with Space Applications. Englewood Cliffs, NJ: Prentice-Hall, 1964, ch. 7.

[5] C. A. Greenhall and R. L. Miller, "Design of a quick-look decoder for the DSN $(7,1 / 2)$ convolutional code," Jet Propulsion Lab., Pasadena, CA, DSN Progress Rep. 42-53, July-Aug. 1979.

[6] K. Y. Liu and J. J. Lee, "An experimental study of the concatenated Reed-Solomon/Viterbi channel coding system performance and its impact on space communications," Jet Propulsion Lab., Pasadena, CA, Pub. 81-58.
[7] G. Lorden, "Procedures for reacting to a change in distribution," $A n n$. Math. Statist., vol. 42, no. 6, pp. 1897-1908, 1971.

[8] R. L. Miller, L. J. Deutsch, and S. A. Butman, "On the error statistics of Viterbi decoding and the performance of concatenated codes," Jet Propulsion Lab., Pasadena, CA, Pub. 81-9.

[9] D. Siegmund, "Importance sampling in the Monte Carlo study of sequential tests," Ann. Statist., vol. 4, pp. 673-684, 1976.

[10] H. G. Tucker, A Graduate Course in Probability. New York: Academic, 1967

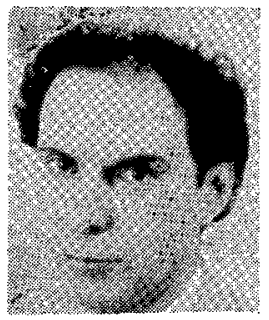

Gary Lorden was born in Los Angeles, CA, in 1941. He received the B.S. degree in mathematics from the California Institute of Technology, Pasadena, in 1962 and the Ph.D. degree in mathematics from Cornell University, Ithaca, NY, in 1966.

After an Assistant Professorship at Northwestern University, Evanston, IL, he returned in 1968 to Caltech, where he is now Professor of Mathematics. His research interests are in mathematical statistics and probability, particularly sequential analysis. $\mathrm{He}$ has been a Consultant to the Jet Propulsion Laboratory, Pasadena, since 1968, and was Visiting Professor of Statistics at the University of California, Berkeley, in 1977-1978.

Dr. Lorden is a Fellow of the Institute of Mathematical Statistics.

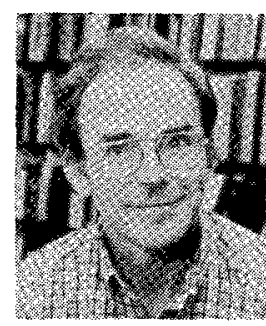

Robert J. McEliece (M'70-SM'81) was born in Washington, DC, in 1942. He received the B.S. and $\mathrm{Ph} . \mathrm{D}$. degrees in mathematics from the California Institute of Technology, Pasadena, in 1964 and 1967, respectively, and attended Trinity College, University of Cambridge, Cambridge, England, during 1964-1965.

From 1963 to 1978 he was employed by the California Institute of Technology's Jet Propulsion Laboratory, where he was Supervisor of the Information Processing Group. From 1972 to 1982 he was Professor of Mathematics and Research Professor at the Coordinated Science Laboratory, University of Illinois, Urbana-Champaign. Since 1982 he has been Professor of Electrical Engineering at Caltech. He is also a Consultant with the Jet Propulsion Laboratory and with Cyclotomics, Inc. His research interests include telecommunications, computer memories, and applied mathematics.

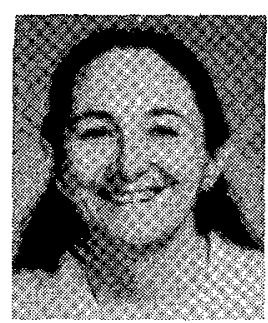

Laif Swanson was born in California on May 13, 1950. She received the B.A. degree in mathematics from the University of California, Irvine, in 1970, and the $\mathrm{Ph} . \mathrm{D}$. degree from the University of California, Berkeley, in 1975.

After six years with the Department of Mathematics, Texas A\&M University, College Station, she joined the Communications Research Section of the Jet Propulsion Laboratory, Pasadena, CA, in 1981. Her interests include coding, information theory, ergodic theory, and probability theory, and she is currently a Visiting Lecturer in the Department of Mathematics, California Institute of Technology, Pasadena. 